\title{
Incidence angle-dependent broadband chiral metamaterial for near- infrared light absorption
}

\author{
JunXING Fan, ${ }^{1,3}$ Dong XIAO 2,3 ,TING LeI, ${ }^{1}$ AND XIAOCONG YUAN ${ }^{1, *}$ \\ ${ }^{1}$ Nano-Photonics Research Center, Shenzhen University, Shenzhen 518060, China \\ ${ }^{2}$ Centre for Biophotonics, Strathclyde Institute of Pharmacy and Biomedical Sciences, University of Strathclyde, Glasgow, G4 ORE, U.K. \\ ${ }^{3}$ These authors contributed equally to this work \\ *xcyuan@szu.edu.cn
}

\begin{abstract}
The ability to spin-selectively absorb circularly polarized light (CPL) plays a critical role in various photonic devices. Here we propose and investigate a broadband chiral metamaterial composed of asymmetric split-ring resonators (SRRs), showing a wide spin-selective absorption band from $950 \mathrm{~nm}$ to $1200 \mathrm{~nm}$ with pronounced circular dichroism (CD) up to $20^{\circ}$. We demonstrate that the broadband absorption spectra originate from induced dual chiral resonance modes. Meanwhile, the two different resonances can be adjusted independently, suggesting great flexibility to the designed chiral absorption band for different purposes. Also, the chiralselective absorption performance is highly dependent on the oblique incident angle due to the extrinsic chirality. The chiral resonance modes can either be enhanced or destructed under oblique incidence. Such angle-dependent broadband chiral metamaterials may find potential applications for spin־orbit communications, chiral detection, polarimetric imaging, and biosensors.
\end{abstract}

\section{Introduction}

Light absorption is of great importance for a variety of photonic devices ranging from sensors, solar cells, and photodetectors. Thanks to the advent of metamaterials, metamaterial absorbers (MAs) with artificial engineered nanostructures have generated huge interests among scientific communities due to their unique properties such as high absorption efficiency (perfect absorption) [1], tunable absorption wavelength from visible light to radiofrequency wave [2], and ultra-thin scale in comparison with traditional bulky materials [3]. Till now, numerous kinds of single- or broad-band MAs have been proposed and found applications in many areas such as energy-harvesting devices [4], biosensors [5], or thermal emitters [6]. However, most of the MAs are insensitive to the circular polarization state of the incident light. In other words, they lack chirality. Chirality refers to an object with its geometry lacking any mirror symmetry plane, which is a pervasive phenomenon and can be found in quartz crystals [7], liquid crystals [8], proteins, and amino acids [9]. One unique feature of chiral structures is the chiroptical effects, i.e. different optical responses to the right or left CPL. Typical chiroptical effects include optical activity and CD [10-14]. Chirality has many important applications in multidisciplinary fields such as chemistry [15], biomedical engineering [16], and pharmacy [17]. In contrast to natural chiral media that have extremely weak chiroptical effects, chiral metamaterials with artificially designed asymmetry unit can obtain pronounced chiroptical effects due to the enhanced plasmonic resonance. Additionally, many exotic chiralityinduced phenomena such as negative refractive index [18], asymmetric transmission [19, 20], and superchiral [21] fields have been demonstrated in chiral metamaterials. In particular, chiral MAs are also proposed and experimentally demonstrated to manipulate the light. Chiral MAs can absorb one particular state CPL while reflecting the other state CPL. Compared to traditional achiral MAs, chiral MAs show great potential in many applications including but not being limited in spinselective detectors, spin lasers, and quantum communications.

Although tremendous efforts have been made to design chiral MAs, the typical absorption spectrum of chiral MAs is narrowband because of the nature of plasmonic resonance. To extend the absorption bandwidth, the general idea is to merge different resonances into one device. In the design of broadband achiral MAs, there are two different routes, one is to design subunits with different resonances in one metamaterial unit cell [22]; the other is to design layer-by-layer stacked structures with different resonances [23]. These ideas are also applied in designing of broadband chiral MAs. For example, Jing et. al [24] have proposed a broadband chiral MAs in the microwave region, in which two different subunits with adjacent resonances are laterally arranged in one period. Therefore, the whole macro unit can obtain a broadband absorption spectrum. However, these methods face intrinsic difficulty when designing chiral MAs in higher frequency regions, especially for optical or infrared waveband. This can be explained below, in achiral MAs, the absorption mechanism is based on the sole magnetic resonance or electric resonance. As structure scales down, different resonances can still keep isolated. However, in chiral MAs, the chirality results from the coupling between the magnetic and electric dipoles. The absorption mechanism is the joint effect of both electric and magnetic resonances. As for chiral MAs working in optical or infrared waveband, their structures are compactly arranged in an ultra-small scale. Different electric or magnetic modes are unavoidable to couple among each other, resulting in the degradation of the absorption bandwidth. Recently, some works have been reported. For example, Alexander et. al [25] have proposed a complex planar dielectric nanostructure with strong intrnsic chirality. Our group [26] has proposed a dielectric nanoarcmetasurface with broadband chiral 
splitters and the structure has been applied in chiral beam splitting, broadband holography. However, the dielectric structure is difficult to achieve broadband chiral absorption due to the character of the ultra-low intrinsic losses of the dielectric metasurface. Therefore, it remains a great challenge to design the broadband chiral MAs.

In this work, a broadband chiral metamaterial absorber (MA) working in near-infrared waveband is

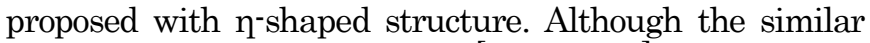
structures have been studied [20,27, 28], the precious structures do not have the broadband absorption performance. Here, we demonstrate that the unit cell of the metamaterial can support the strong dual chiral resonance modes without degradation, resulting in nearperfect broadband absorption for one specified sate of CPL. The resonant wavelength of different chiral resonance modes can be separately adjusted by changing the structure parameters. The underlying mechanism of the chiral resonance modes is also analyzed. Furthermore, we show that our proposed chiral MA has angle-dependent absorption behavior for oblique incident light. Our proposed design may find applications in exotic devices for chirality detection, holographic imaging, and quantum communications.

\section{Design and simulation}

The origin of chirality is the coupling effect between the electric and magnetic dipoles. To obtain pronounced chiroptical effects, one should make sure that both electric and magnetic modes are coexisted and efficiently coupled within the plasmonic structures. To this end, the unit of our proposed metamaterial absorber is designed to be a $\eta^{-}$ shaped split ring resonator (nSRR) illustrated in Fig 1. (a). The detailed design of the proposed chiral MA is depicted in Fig. 1 (b) and (c). The nSRRs are aligned on a thick golden backplane separated by a silica spacer. Another silica upper cover layer is added to ensure that the nSRR layer has a homogeneous dielectric surrounding. The nSRR layer and the backplane form a Fabry-Perot-like cavity, which is known as the MIIM structure and can support magnetic dipole in the dielectric layer. On the other hand, the nSRR structure can excite electric dipole within its plane. Therefore, it is possible to obtain a strong coupling between two different dipoles. Figure 1 (c) shows

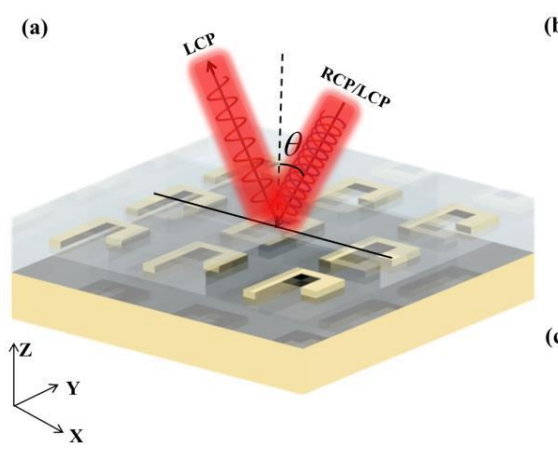

the top view of a single unit of the $\eta \mathrm{SRR}$ structure, the two arms with different lengths and widths break the geometric symmetry, leading to a chiroptical response to the incident CPL with the opposite spin state.

The finite-difference time-domain (FDTD) method (Lumerical) is utilized to investigate the optical response and physical mechanism. The single unit structure is simulated with periodic boundary condition applied on $\mathrm{x}^{-}$ and $\mathrm{y}^{-}$directions while perfectly matched layer (PML) on $\mathrm{z}$-direction. The dispersive dielectric parameter of the gold is depicted by the Drude model and the refractive index of the silica is 1.45. The thickness of the backplane is $200 \mathrm{~nm}$, which is larger than the skin depth of the gold in the wavelength region of interest. The RCP and LCP lights are incidence the chiral MA with incident angle $\theta$. Since the backplane is thick enough, the transmittance is zero and the absorptivity for RCP and LCP lights can be calculated by:

$$
A_{R C P / L C P}=1-R_{R C P / L C P}
$$

where $A_{R C P L C P}$ and $R_{R C P L C P}$ denote the absorptivity and reflectance for RCP and LCP incident lights. The corresponding CD spectrum is defined as [29]:

$$
\theta(\mathrm{deg})=\frac{180}{\pi} \tan ^{-1}\left(\frac{\sqrt{R_{R C P}}-\sqrt{R_{L C P}}}{\sqrt{R_{R C P}}+\sqrt{R_{L C P}}}\right) \approx 33 \triangle A
$$

where $\triangle A=A_{R C P}-A_{L C P}$ is the difference in the absorptivity of RCP and LCP lights.

Figure 2(a) shows the calculated absorption spectra for both RCP and LCP normal incident lights $\left(\theta=0^{\circ}\right)$ at the range from $800 \mathrm{~nm}$ to $1400 \mathrm{~nm}$. A significant difference can be observed in absorptivity for RCP and LCP lights from $870 \mathrm{~nm}$ to $1400 \mathrm{~nm}$. The absorption spectra of RCP light show a broad and flat absorption band with absorbance larger than $90 \%$ from $950 \mathrm{~nm}$ to $1200 \mathrm{~nm}$. In contrast, the average absorbance for LCP keeps a quit low rate of about $40 \%$ and has no dramatic change throughout the wavelength range. The corresponding $\mathrm{CD}$ spectrum is shown in Fig. 2 (b). The resulted CD reaches around $20^{\circ}$ in a wide wavelength range, demonstrating pronounced chirality for our proposed MA. The strong chirality is attributed to different plasmonic resonance modes under RCP illumination.

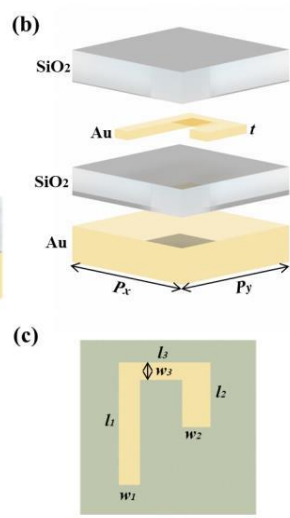

Fig. 1 (a) Schematic of the chiral metamaterial for the RCP or LCP incident lights. The incident angle $\theta$ is the angle with the z-axis; (b), (c) The decomposed structures and the top view of the metamaterial with the important structural parameters: $t=24 \mathrm{~nm}, h=170 \mathrm{~nm}, m$ $=200 \mathrm{~nm}, l_{1}=353 \mathrm{~nm}, l_{2}=184 \mathrm{~nm}, l_{3}=254 \mathrm{~nm}, w_{1}=56 \mathrm{~nm}, w_{2}=74 \mathrm{~nm}, w_{3}=55 \mathrm{~nm}, P_{x}=P_{y}=400 \mathrm{~nm}$. 


\section{Results and discussion}

To understand the broadband chiral-selective absorption behavior, the dispersive absorption spectra of RCP under different structural parameters are also calculated. For our proposed structure, the chiral-selective absorption properties are mainly determined by six geometric parameters $\left(l_{1}, W_{1}, l_{2}, W_{2}, l_{3}, W_{3}\right)$ as denoted in Fig. 1 . The results are shown in Fig.3 (a)-(f), in which one structure parameter changes while other parameters are fixed the same as that in Fig. 1. Two independent absorption bands contribute to the whole absorption spectra. By changing the parameters, they undergo different resonance shifts. Interestingly, the shorter resonant wavelength is mainly decided by the length of the long arm and the width of the short arm $\left(l_{1}, W_{2}\right)$ while the longer resonant wavelength is mainly decided by the length of the short arm and the width of the long arm $\left(l_{2}, w_{1}\right)$. Additionally, both resonant wavelengths are affected by the width and length of the connection arm. As depicted by the white lines in Fig.3 (a)-(f), the wavelengths of the resonant peaks have linear relationships with the structural parameters within the investigated wavelength range. Therefore, the two different resonances can be precisely adjusted independently, offering great flexibility to the designed absorption band for various purposes.

The induced magnetic and electric field distributions at the two resonant wavelengths for the RCP and LCP normal incident lights are further calculated to explore the underlying physical mechanism. The results are shown in Fig. 4, where the normalized electric and magnetic fields lie on the interface between the patterned $\mathrm{Au}$ layer and the $\mathrm{SiO}_{2}$ spacer. The broadband chiralselective absorption is attributed to Ohmic loss of the

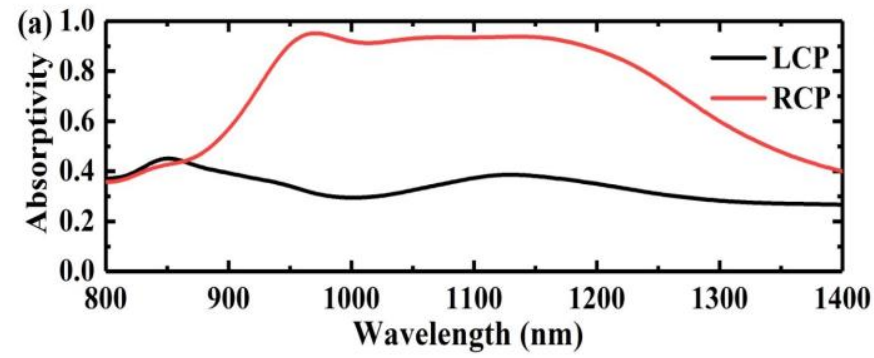

combined different plasmonic resonance modes induced by charge oscillation at the surfaces of the metamaterial. The electric and magnetic fields induced by RCP illumination are more pronounced than that of LCP illumination, leading to strong RCP light absorption. Compared with the achiral MIM structure, the electronic and magnetic field distributions in the proposed metamaterial show similar properties in electronic and magnetic dipole resonances [30]. For RCP incident light, it is clear to observe that the electric fields are remarkably enhanced at some corners, indicating there is a large amount of charges accumulation. This is the main feature of electric dipole resonance since the charge accumulation would generate the strong electric potential, leading to the electric dipole moment. The dipole moments, in turn, drive the antiparallel electric current in the gold patch and substrate. This induced current form a closed circuit together with the displacement current in the dielectric spacer, leading to a strong magnetic dipole in the spacer layer. Therefore, it's seen that the magnetic field distributions are located on the edges of the gold structure as shown in Fig.4 (b) and (d), which are also similar to the magnetic field distributions in achiral MIM structure. For incident LCP light, only weak electric and magnetic field distributions are observed for both resonant wavelengths, showing no effective resonances. It is demonstrated that the resonance modes are highly dependent on the spin state of the incident light. Also, the field distributions at $970 \mathrm{~nm}$ and $1140 \mathrm{~nm}$ are similar. For shorter resonant wavelength, the "wave node" of both electric and magnetic field distributions are denser compared with that of longer the resonant wavelength, indicating that it's a higherorder mode.

Fig. 2 (a) Absorption spectra of the metamaterial; (b) CD spectrum with the RCP and LCP normal incident lights.
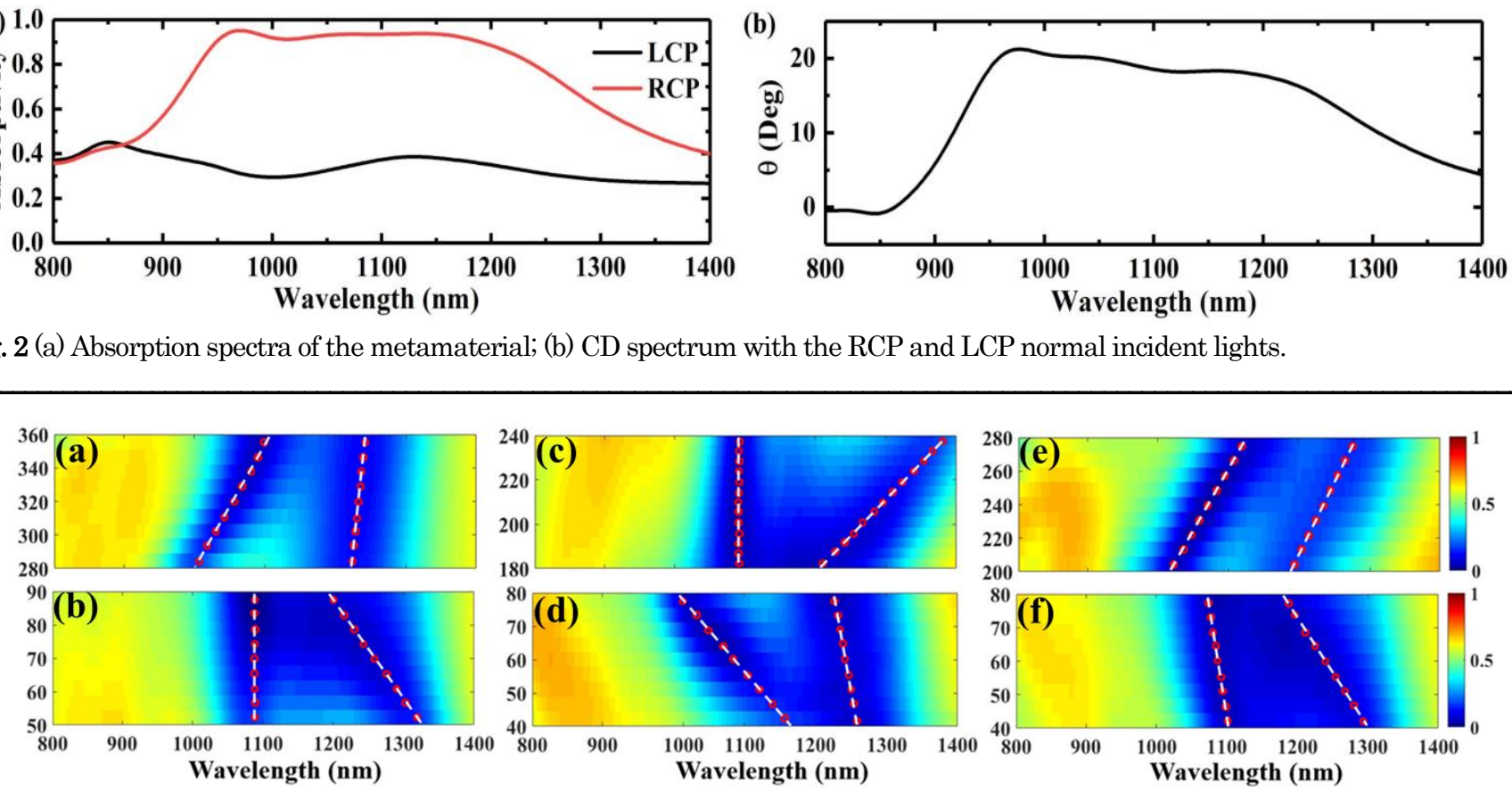

Fig. 3 Reflection spectra of the metamaterial with changing structural parameters for the RCP incident light. (a) $l_{1}$; (b) $W_{1}$; (c) $l_{2}$; (d) $W_{2}$; (e) $l_{3}$; (f) $W_{3}$. The red dots are the calculated wavelengths of the resonant peaks and the white lines are the fitting curve of the resonances. 
(a)

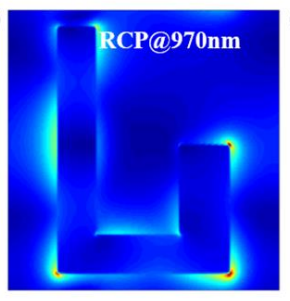

(b)

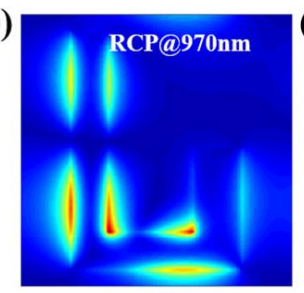

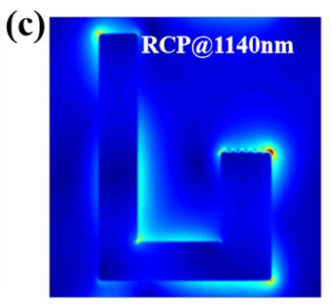

(d)

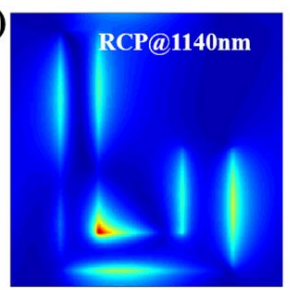

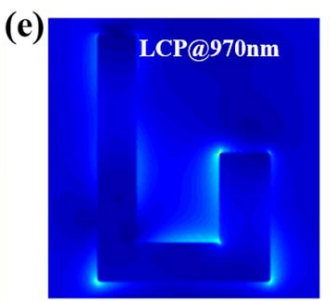

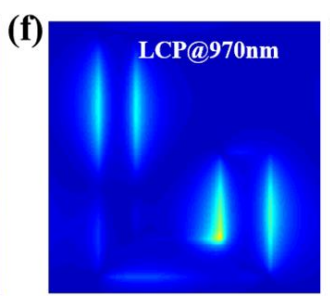

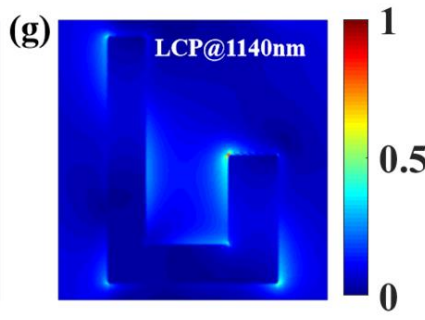

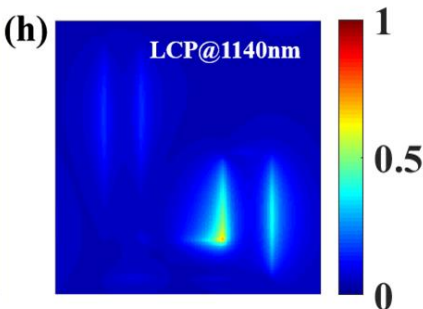

Fig. 4 The normalized electric and magnetic distributions at different resonant peaks for the RCP and LCP incident lights. (a), (c), (e) and (g) The normalized electric distributions; (b), (d), (f) and (h) The normalized magnetic distributions.

In previous proposed achiral MAs, one remarkable light absorption performance is the angle insensitive property. The MAs keep near-perfect absorptivity at a wide range of oblique incidence angle. However, the situation becomes complex in chiral metamaterial absorbers. For the normal CPL incidence, the chirality of the metamaterial discussed above is associated with its intrinsic chirality resulting from the coupling of both electric and magnetic dipoles. For the oblique CPL incidence, the mutual orientation of the wave propagation direction and the metamaterial would lead to the tilt of the exciting electric and magnetic dipoles, resulting in extrinsic chirality. Extrinsic chirality has been observed even in achiral planner metamaterials [31, 32]. To the best of our knowledge, no work has been carried out to evaluate the effect of extrinsic chirality on the absorption characteristics. Therefore, it's worth investigating the chiral-selective absorption characteristics under oblique angles. Figure 5 shows the absorption spectra under LCP and RCP lights with incident angles from $\pm 5^{\circ}$ to $\pm 35^{\circ}$. Interesting phenomena can be observed for both positive and negative incidence angle $\theta$. When $\theta$ varies from $5^{\circ}$ to $35^{\circ}$, the broadband absorption spectra keep well with strong absorptivity for RCP incident light. The resonance with longer wavelength shows a redshift while the resonance with shorter wavelength almost stays unchanged. Meanwhile, the absorption spectra for RCP incident light nearly have no change, indicating that charity maintains well for positive incidence angles. On the other hand, when $\theta$ varies from $-5^{\circ}$ to $-35^{\circ}$, resonance with a longer wavelength not only shows a redshift but also experiences a significant decrease in absorptivity. At the same time, the absorptivity for LCP incident light increases from around 0.3 to 0.7 . It is easy to see that the chirality is near to zero at $\lambda \approx 1260 \mathrm{~nm}$, showing that the chirality of resonance with longer wavelength vanishes at a negative incident angle. Hence, the broadband absorption spectra no longer maintain. In contrast, the chirality of resonance with shorter wavelengths is unchanged.

The significantly enhanced chirality of the proposed metasurface arises from the coupling of the excited magnetic and electric dipole moments, forming a combined charge-oscillation eigenmode. This enhanced chirality can be quantitatively expressed by [33-35]:

$$
C=-\frac{\varepsilon_{0} \omega}{2} \operatorname{Im}\left(\vec{E}^{*} \cdot \vec{B}\right)
$$

where $\varepsilon_{0}$ is the dielectric permittivity in vacuum and $\omega$ is the angular frequency. $\vec{E}$ and $\vec{B}$ are the complex electric and magnetic field vectors, respectively. The asterisk * denotes the complex conjugate. The pseudoscalar $C$ characterizes the chirality of light. For instance, $C_{L P L}=0$ is for linear polarized light propagating in free space and $C_{C P L}=\varepsilon_{0} \omega / 2 c_{0}\left|E^{2}\right|$ is for CPL propagating in free space, where $c_{0}$ is the vacuum velocity of light. The enhanced chirality distribution maps under $\pm 35^{\circ} \mathrm{LCP} / \mathrm{RCP}$ incident light are further analyzed. As shown in Fig. 6, for RCP incident light, the pronounced enhanced chirality mainly occurs at the structure's corners and edges. As a comparison, for LCP incident light, the value of enhanced absorption spectrum shows a significant decrease, especially for the resonance at $1240 \mathrm{~nm}$ in Fig. 5(g). Therefore, the extrinsic chirality could either contribute to or destruct the chirality of the metamaterial. Similar results can also be observed for LCP light under oblique incident angles. The chiral MAs have a weak absorption capability for LCP under normal incident light. However, as shown in Fig. 6(h), a strongly enhanced chirality occurs at the short arm of the metamaterial, resulting in an increase of absorption in Fig. 5(h). The enhanced chirality distribution maps well explain the variation of the chiral absorption under oblique incident light. 

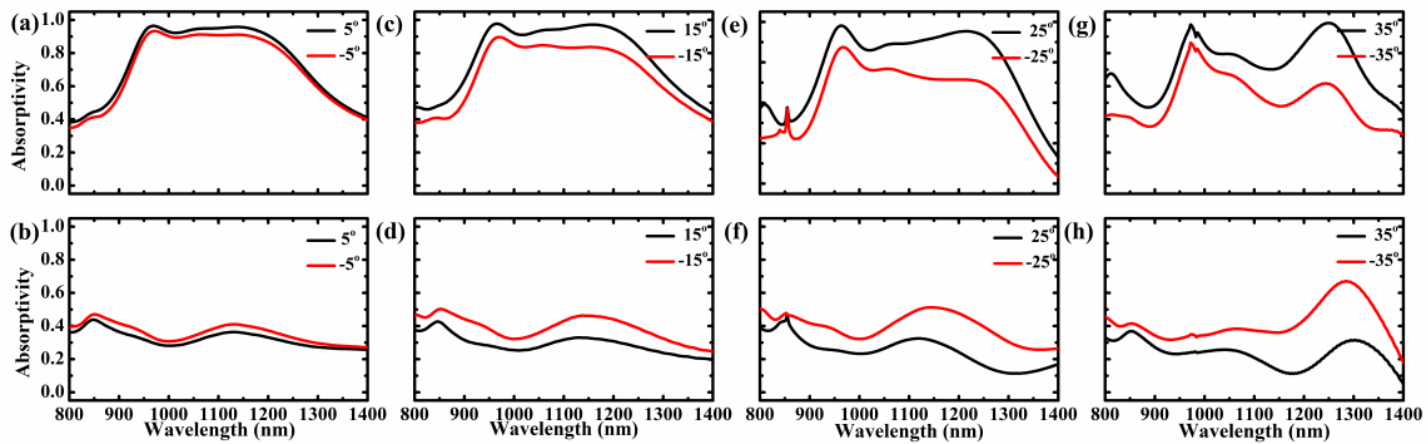

Fig. 5 Absorption spectra of the metamaterial for the RCP and LCP incident lights with incident angles from $\theta= \pm 5^{\circ}$ to $\pm 35^{\circ}$ in steps of $10^{\circ}$. (a) RCP, $\pm 5^{\circ}$; (b) LCP, $\pm 5^{\circ}$; (c) RCP, $\pm 15^{\circ}$; (d) LCP, $\pm 15^{\circ}$; (e) RCP, $\pm 25^{\circ}$; (f) LCP, $\pm 25^{\circ}$; (g) RCP, $\pm 35^{\circ}$; (h) LCP, $\pm 35^{\circ}$.
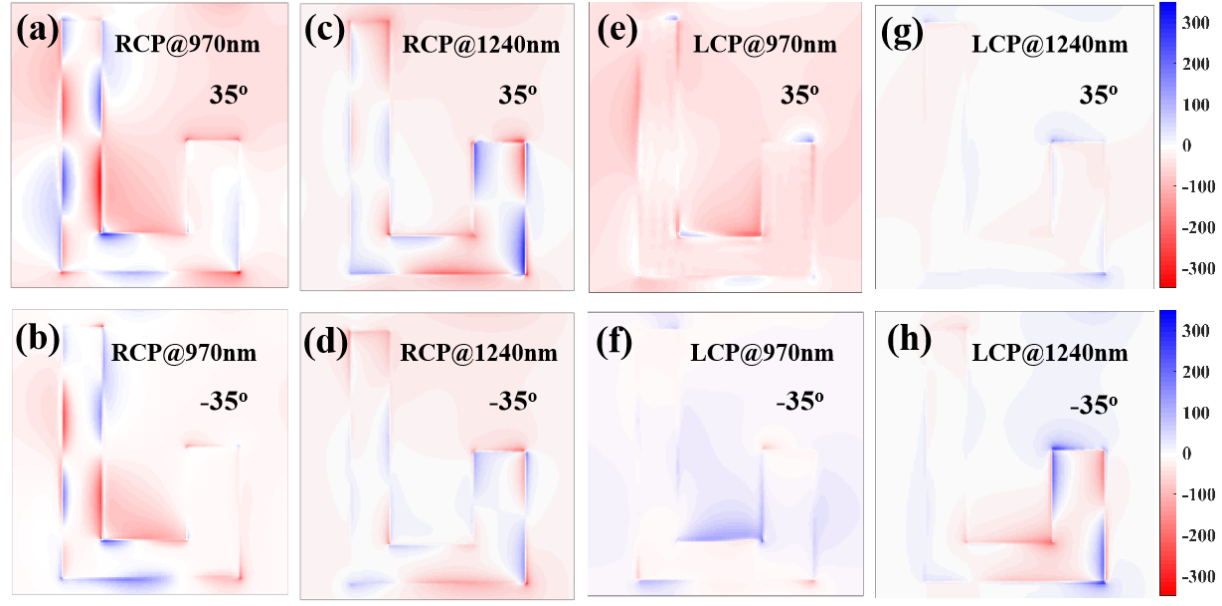

Fig. 6 Enhanced optical chirality of the metamaterial for the RCP and LCP incident lights at two resonant frequencies with the tilt angles $\pm 35^{\circ}$.

Table 1 Some key performance metrics related to the broadband chiral metamaterial; in the different references. $\lambda_{c}:$ Central wavelength; FWHM : Full width at half maximum; $\Delta T / R:\left|T_{R C P}-T_{L C P}\right| /\left|R_{R C P}-R_{L C P}\right| ; H W H M / \lambda_{c}:$ Bandwidth.

\begin{tabular}{|l|l|l|l|l|l|}
\hline Ref. & $\lambda_{c}(\mu \mathrm{m})$ & $F W H M / \lambda_{c}$ & $\Delta T / R$ & Number of cell & Dimension \\
\hline$[35]$ & $5.5 \mu \mathrm{m}$ & $72 \%$ & 0.7 & 1 & 3-dimension \\
\hline$[36]$ & $0.75 \mu \mathrm{m}$ & $15 \%$ & 0.2 & 1 & 3-dimension \\
\hline$[37]$ & $0.98 \mu \mathrm{m}$ & $20 \%$ & 0.35 & 1 & 3-dimension \\
\hline$[38]$ & $1.6 \mu \mathrm{m}$ & $31 \%$ & 0.6 & 6 & 2-dimension \\
\hline Our's & $1.1 \mu \mathrm{m}$ & $35 \%$ & 0.55 & 1 & 2-dimension \\
\hline
\end{tabular}

Some important performance metrics related to broadband chirality metamaterial from the visible region to the middle infrared region are summarized in table 1. For the reference 33 in Table 1, we can clearly observe that the helix structure of metamaterial can achieve ultra-bandwidth chirality with a higher $\Delta T$ at the middle infrared wavelengths. The helix structure is a complicated three-dimension geometry, which leads to significant fabrication challenges, especially at visible wavelengths.
Meanwhile, the helix structure for reference 34 cannot achieve the ultra-bandwidth chirality at the visible wavelengths with the decrease of the helix structure. For reference 35 , we can clearly observe that the bandwidth and $\Delta T$ are not a highlight. As shown in reference 36 , it can be seen that the metamaterial has a dramatical advantage in the bandwidth and $\Delta R$, but the number of cell is six, which leads to an increase of the structure size. After comprehensive consideration, our structure in the 
near infrared region is a good choice to achieve the broadband chiral absorption with the dramatical chiroptical effects.

\section{Conclusion}

In summary, we have proposed and demonstrated the nSRR-shaped chiral MA with a broadband absorption from $950 \mathrm{~nm}$ to $1200 \mathrm{~nm}$ in near-infrared waveband and circular dichroism up to $20^{\circ}$. We have demonstrated that the unit cell of the metamaterial can support strong different resonance modes without degradation, resulting in near-perfect broadband absorption for one specified sate of CPL. Meanwhile, in our proposed structure, the different resonances can be adjusted independently by changing the structure parameters, offering great flexibility to the designed absorption band for various purposes. Furthermore, our proposed chiral MA has angle-dependent absorption behavior for oblique incident light due to the extrinsic chirality. Therefore, our proposed design may find applications in exotic devices for chirality detection, holographic imaging, and quantum communications.

Funding. National Natural Science Foundation of China (U1701661，61427819，11774240，11604218); Leading Talents of Guangdong Province Program (00201505); Science and Technology Innovation Commission of Shenzhen (KQTD2015071016560101, KQTD2017033011 0444030, KQJSCX20170727100838364); Natural Science Foundation of Guangdong Province, China (2016A030312010).

\section{Disclosures.}

The authors declare no conflicts of interest.

\section{REFERENCES}

1. N. I. Landy, S. Sajuyigbe, J. J. Mock, D. R. Smith, and W. J. Padilla, "Perfect Metamaterial Absorber," Phys. Rev. Lett. 100, 207402-207405 (2008).

2. D. Shrekenhamer, W. C. Chen, and W. J. Padilla, "Liquid Crystal Tunable Metamaterial Absorber," Phys. Rev. Lett. 110, 177403-177407 (2013).

3. Z. Jingjing, J. B. Pendry, and L. Yu, "Transformation optics from macroscopic to nanoscale regimes: a review," Adv. Photonics 1, 014001-014015 (2019).

4. O. M. Ramahi, T. S. Almoneef, M. Alshareef, and M. S. Boybay, "Metamaterial particles for electromagnetic energy harvesting," Appl. Phys. Lett. 101, 5 (2012).

5. S. RoyChoudhury, V. Rawat, A. H. Jalal, S. N. Kale, and S. Bhansali, "Recent advances in metamaterial split-ring-resonator circuits as biosensors and therapeutic agents," Biosens. Bioelectron. 86, 595-608 (2016).
6. J. J. Greffet, R. Carminati, K. Joulain, J. P. Mulet, S. P. Mainguy, and Y. Chen, "Coherent emission of light by thermal sources," Nature 416, 61-64 (2002).

7. R. M. Hazen, and D. S. Sholl, "Chiral selection on inorganic crystalline surfaces," Nat. Mater. 2, 367-374 (2003).

8. J. Kobashi, H. Yoshida, and M. Ozaki, "Planar optics with patterned chiral liquid crystals," Nat. Photonics 10, 389-+ (2016).

9. H. Berman, K. Henrick, H. Nakamura, and J. L. Markley, "The worldwide Protein Data Bank (wwPDB): ensuring a single, uniform archive of PDB data," Nucleic Acids Res. 35, D301-D303 (2007).

10. Y. Cheng, F. Chen, and H. Luo, "Multi-band giant circular dichroism based on conjugated bilayer twisted-semicircle nanostructure at optical frequency," Phys. Rev. A. 384, 126398 (2020).

11. F. Fang, Y. Cheng, and H. Liao, "Giant optical activity and circular dichroism in the terahertz region based on bi-layer Y-shaped chiral metamaterial," Optik 125, 6067-6070 (2014).

12. Y. Z. Cheng, Y. L. Yang, Y. J. Zhou, Z. Zhang, X. S. Mao, and R. Z. Gong, "Complementary Yshaped chiral metamaterial with giant optical activity and circular dichroism simultaneously for terahertz waves," J. Modern Opt. 63, 1675-1680 (2016).

13. Y. Z. Cheng, M. L. Huang, H. R. Chen, Y. J. Zhou, X. S. Mao, and R. Z. Gong, "Influence of the geometry of a gammadion stereo-structure chiral metamaterial on optical properties," J. Modern Opt. 64, 1487-1494 (2017).

14. Y. Z. Cheng, H. Lu, F. Chen, X. S. Mao, and R. Z. Gong, "Photo-excited switchable broadband linear polarization conversion via asymmetric transmission with complementary chiral metamaterial for terahertz waves," Osa Continuum 2, 2391-2400 (2019).

15. C. Kelly, R. Tullius, A. J. Lapthorn, N. Gadegaard, G. Cooke, L. D. Barron, A. S. Karimullah, V. M. Rotello, and M. Kadodwala, "Chiral Plasmonic Fields Probe Structural Order of Biointerfaces," J. Am. Chem. Soc. 140, 8509-8517 (2018).

16. C. Jack, A. S. Karimullah, R. Leyman, R. Tullius, V. M. Rotello, G. Cooke, N. Gadegaard, L. D. Barron, and M. Kadodwala, "Biomacromolecular Stereostructure Mediates Mode Hybridization in Chiral Plasmonic Nanostructures," Nano Lett 16, 5806-5814 (2016).

17. E. B. G. Pályi, C. Zucchi, and L. Caglioti, "Advances in BioChirality," (1999).

18. S. Zhang, Y. S. Park, J. Li, X. Lu, W. Zhang, and X. Zhang, "Negative Refractive Index in Chiral Metamaterials," Phys. Rev. Lett. 102, 023901-023904 (2009). 
19. Shan, $\mathrm{Wu}, \mathrm{Su}, \mathrm{Xu}, \mathrm{Yi}$, Zhang, Yanning, Jianjuan, Jiang, and Qianjin, "Asymmetric transmission and optical rotation of a quasi-3D asymmetric metallic structure," Opt. Lett. 39, 64266429 (2014).

20. B. Tang, Z. Li, E. Palacios, Z. Liu, S. Butun, and K. Aydin, "Chiral-Selective Plasmonic Metasurface Absorbers Operating at Visible Frequencies," IEEE Photon. Technol. Lett. 29, 295298 (2017).

21. E. Hendry, T. Carpy, J. Johnston, M. Popland, R. V. Mikhaylovskiy, A. J. Lapthorn, S. M. Kelly, L. D. Barron, N. Gadegaard, and M. Kadodwala, "Ultrasensitive detection and characterization of biomolecules using superchiral fields," Nat. Nanotechnol. 5, 783-787 (2010).

22. Y. Wen, W. Ma, J. Bailey, G. Matmon, X. Yu, and G. Aeppli, "Planar broadband and high absorption metamaterial using single nested resonator at terahertz frequencies," Opt. Lett. 39, 1589-1592 (2014).

23. M. Amin, M. Farhat, and H. Bagci, "An ultrabroadband multilayered graphene absorber," Opt. Express 21, 29938-29948 (2013).

24 L. Jing, Z. Wang, Y. Yang, B. Zheng, Y. Liu, and $\mathrm{H}$. Chen, "Chiral metamirrors for broadband spin-selective absorption," Appl. Phys. Lett. 110, 231103 (2017).

25. A. Y. Zhu, W. T. Chen, A. Zaidi, Y. W. Huang, M. Khorasaninejad, V. Sanjeev, C. W. Qiu, and F. Capasso, "Giant intrinsic chiro-optical activity in planar dielectric nanostructures," Light Sci. Appl. 7, 17158-17158 (2018).

26. D. Wang, Y. Hwang, Y. Dai, G. Si, S. Wei, D. Y. Choi, D. E. Gomez, A. Mitchell, J. Lin, and X. Yuan, "Broadband High-Efficiency Chiral Splitters and Holograms from Dielectric Nanoarc Metasurfaces," Small 15, 1-8 (2019).

27. A. B. Khanikaev, N. Arju, Z. Fan, D. Purtseladze, F. Lu, J. Lee, P. Sarriugarte, M. Schnell, R. Hillenbrand, M. A. Belkin, and G. Shvets, "Experimental demonstration of the microscopic origin of circular dichroism in two-dimensional metamaterials," Nat. Commun. 7, 12045-12045 (2016).

28. W. Li, Z. J. Coppens, L. V. Besteiro, W. Wang, A. O. Govorov, and J. Valentine, "Circularly polarized light detection with hot electrons in chiral plasmonic metamaterials," Nat. Commun. 6, 83798379 (2015).

29. Y. Cheng, H. Chen, J. Zhao, X. Mao, and Z. Cheng, "Chiral metamaterial absorber with high selectivity for terahertz circular polarization waves," Opt. Mater. Express 8, 1399-1409 (2018).

$30 . \quad J$. M. Hao, J. Wang, X. L. Liu, W. J. Padilla, L. Zhou, and M. Qiu, "High performance optical absorber based on a plasmonic metamaterial," App. Phys. Lett. 96, 3 (2010).

31. E. Plum, V. A. Fedotov, and N. I. Zheludev, "Optical activity in extrinsically chiral metamaterial," Appl. Phys. Lett. 93, 191911 (2008).

32. E. Plum, X. X. Liu, V. A. Fedotov, Y. Chen, D. P. Tsai, and N. I. Zheludev, "Metamaterials: optical activity without chirality," Phys. Rev. Lett. 102, 113902-113905 (2009).

33. Y. Tang, and A. E. Cohen, "Optical Chirality and Its Interaction with Matter," Phys. Rev. Lett. 104, 163901 (2010).

34. M. Schäferling, D. Dregely, M. Hentschel, and H. Giessen, "Tailoring Enhanced Optical Chirality: Design Principles for Chiral Plasmonic Nanostructures," Phys. Rev. X 2, 031010-031018 (2012).

35. D. Xiao, Y. J. Liu, S. Yin, J. Liu, W. Ji, B. Wang, D. Luo, G. Li, and X. W. Sun, "Liquid-crystalloaded chiral metasurfaces for reconfigurable multiband spin-selective light absorption," Opt Express 26, 25305-25314 (2018).

36. M. Esposito, V. Tasco, M. Cuscunà, F. Todisco, A. Benedetti, I. Tarantini, M. D. Giorgi, D. Sanvitto, and A. Passaseo, "Nanoscale 3D Chiral Plasmonic Helices with Circular Dichroism at Visible Frequencies," ACS Photonics 2, 105-114 (2014).

37. S. E. Mun, J. Hong, J. G. Yun, and B. Lee, "Broadband circular polarizer for randomly polarized light in few-layer metasurface," Sci Rep 9, 2543 (2019).

38. L. Ouyang, D. Rosenmann, D. A. Czaplewski, J. Gao, and X. Yang, "Broadband infrared circular dichroism in chiral metasurface absorbers," Nanotechnology 31, 295203-295208 (2020). 